
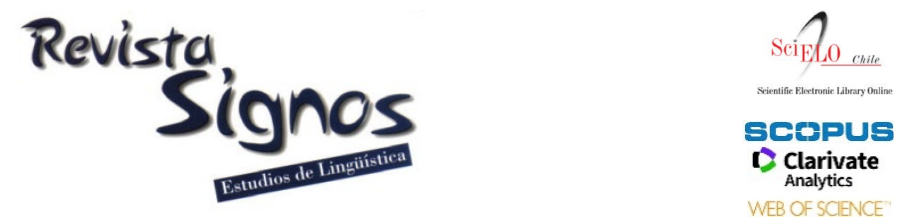

\title{
El estilo de habla en el discurso directo como estrategia para la construcción del género en el monólogo humorístico ${ }^{1}$
}

\author{
Speech style in direct discourse as a strategy for gender \\ construction in stand-up comedy
}

\author{
Esther Linares Bernabéu \\ UNIVERSIDAD DE ALICANTE \\ ESPAÑA \\ esther.linares@ua.es
}

Recibido: 16-VII-2018 / Aceptado: 22-IV-2019

DOI: $10.4067 /$ S0718-09342020000100123

\section{Resumen}

La presente investigación explora el papel del discurso humorístico planificado en la construcción de la identidad de género. El objetivo principal es explorar las distintas formas en las que las humoristas españolas hacen uso del estilo de habla femenino y masculino, a través del discurso directo, con el objeto de romper con los roles sexuales y subvertir las nociones heteronormativas de masculinidad y feminidad. Partimos de la hipótesis de que, en el discurso humorístico, el estilo de habla femenino y masculino se usan con independencia del sexo para conseguir unos determinados efectos cómicos y sociales. Con el objetivo de verificar tal conjetura, este estudio recoge un corpus de 10 monólogos humorísticos representados en salas y teatros españoles durante los años 2017 y 2018. La transcripción y segmentación de dichas muestras nos ha permitido examinar la función de los estilos de habla en el discurso directo y su relación con los estilos humorísticos propuestos por Martin, Puhlik-Doris, Larsen, Gray y Weir (2003). $\mathrm{El}$ análisis de los efectos conseguidos en relación con la construcción y deconstrucción de la identidad de género, así como el estudio de los elementos lingüísticos y paralingüísticos empleados para desencadenar el efecto cómico, nos ha permitido comprobar que el uso de un estilo de habla marcado por una determinada identidad de género es un instrumento discursivo que puede implicar tanto la ruptura de determinados roles sexuales como el refuerzo de tópicos y estereotipos de género.

Palabras Clave: Humor, género, monólogo, discurso directo, estilo de habla. 


\begin{abstract}
This research examines the role humorous planned discourse plays in the construction of gender identity. The main aim is to explore some of the distinct ways in which Spanish female stand-up comedians use humor language and gendered speech styles in direct discourse to deconstruct their imposed gender roles and subvert the heteronormative notions of femininity and masculinity. We depart from the hypothesis that speech styles are used regardless the sex in order to cause certain effects both comical and social. So as to corroborate this idea, our study gathers a corpus which comprises 10 monologues by Spanish stand-ups, performed in Spanish theatres and pubs in the span of time of 2017 and 2018. These monologues were transcribed and divided into humorous sequences in order to examine the main function of the different speech styles and their relationship with the humor styles proposed by Martin Martin, Puhlik-Doris, Larsen, Gray and Weir (2003). The analysis of the effects obtained in relation to the construction and deconstruction of gender identity as well as the account of the most prominent linguistic elements employed, have allowed us to prove that the use of gendered speech styles is a discourse device not only for breaking with sex roles but also for reinforcing and perpetuation some gender clichés.
\end{abstract}

Key Words: Humor, gender, stand-up comedy, direct discourse, speech style.

\title{
INTRODUCCIÓN
}

El humor es un hecho pragmático que ha sido abordado desde diversas perspectivas a lo largo de la historia. Su ubicuidad en todos los ámbitos de nuestro día a día ha llevado a que este fenómeno sea estudiado por ciencias tan dispares como la filosofía, la antropología, la psicología, la etnografía o la lingüística. Por medio de estas ramas, se ha comprobado que la percepción, comprensión y producción del humor difieren atendiendo a variables como la edad, la cultura, el nivel social, la orientación sexual o el género. Este último factor será el que reciba nuestra atención en mayor medida, aunque no por ello vayamos a descuidar el resto de las variables.

Es evidente que el género, como categoría social dinámica, condiciona la mayoría de aspectos de nuestra vida diaria, incluida la comunicación. De hecho, el habla refleja, a la hora de transmitir un mensaje, nuestra identidad de género a través del estilo, el registro y los elementos lingüísticos que seleccionamos, de forma consciente o no. De acuerdo con Chiaro y Baccolini (2014: 1):

"Gender conditions the most minute details of our lives, possible more than our age, our social background, and our ethnicity, and thus, it stands to reason that the way we "do" humor may also, in some way, be accordingly gendered".

Así pues, las líneas anteriores nos llevan a que nos planteemos los siguientes interrogantes: ¿Es posible reconocer la manifestación del género en el discurso humorístico?, ¿qué estrategias narrativas y elementos lingüísticos del humor verbal colaboran en la construcción de la identidad de género?, ¿podemos identificar diferentes estilos de habla marcados por el género en el monólogo humorístico?, y 
¿qué efecto perlocutivo tiene el uso de estos estilos de habla? A fin de resolver estos interrogantes, estudiaremos los procesos de producción y actuación del discurso humorístico a través de 10 monólogos representados por humoristas españolas. Nuestro corpus nos permitirá determinar de qué forma se manifiesta el género, tanto normativo como subversivo, en este tipo de discurso oral planificado.

De forma más concreta, el objetivo de este trabajo es el de explorar, desde la perspectiva discursiva del género, de qué manera las humoristas españolas construyen su identidad a través del humor. Para ello, examinaremos el uso del discurso directo como estrategia empleada por las cómicas para mostrar un determinado estilo humorístico y, en ocasiones, también para reproducir un estilo de habla marcado por rasgos del género femenino o masculino heteronormativo. Asimismo, estudiaremos los principales recursos lingüísticos y extralingüísticos, es decir, los indicadores y marcas humorísticas (Ruiz Gurillo, 2012, 2014) empleados durante las diferentes intervenciones del discurso directo, con el objetivo de identificar los principales efectos que se generan con su uso. Siguiendo la propuesta del grupo GRIALE, entendemos por indicadores humorísticos todos aquellos elementos lingüísticos, ya sean léxicos, sintácticos o fonéticos, que son humorísticos en un determinado contexto y contribuyen a desencadenar el efecto cómico. Mientras que, por su parte, las marcas serían procedimientos prosódicos, kinésicos y tipográficos que ayudan a inferir el humor (Ruiz Gurillo, 2012, 2014).

Partimos de la convicción de que la noción de género está sujeta al uso del lenguaje ${ }^{2}$, por lo que su representación dependerá de los elementos lingüísticos o de la forma en que se utilice el lenguaje en un determinado contexto humorístico. Esta idea se apoya en la teoría constructivista propuesta por la filósofa Judith Butler (1990, 1993) quien argumenta que el género se construye social y culturalmente, es decir, se trata de un constructo social de carácter fluido, que se forma o construye de acuerdo con nuestras acciones dentro de una determinada comunidad ${ }^{3}$ y que, por ende, se reflejará en nuestra habla, como demostraremos en este trabajo.

Por otro lado, seguiremos los postulados de Gal (1995) y Hall (1995), cuando analicemos los estilos de habla en el discurso directo. Según estos autores, el estilo de habla marcado por el género masculino o femenino heterornormativo es independiente del sexo del hablante (Gal, 1995; Hall, 1995; Cameron, 1998), por lo que nuestra hipótesis será que el estilo de habla marcado por el género femenino o masculino es empleado por las humoristas con independencia de su sexo, en aras de conseguir un determinado efecto humorístico y social, como pueda ser el de desafiar el statu quo o el de reforzar lo establecido por el patriarcado. En este sentido, nuestro trabajo rompe con los estudios esencialistas precedentes que han analizado el discurso y el género desde la dualidad biológica del sexo. 
Por consiguiente, en las páginas que siguen a continuación, estableceremos el marco teórico sobre el que se asentará la investigación acerca de la construcción de la identidad de género en el humor y, más concretamente, en el monólogo como discurso planificado (apartado 2). Seguidamente, expondremos los instrumentos metodológicos que hemos empleado para la recogida del corpus y la extracción de los datos (apartado 3). El análisis cuantitativo y cualitativo, junto con la discusión de los resultados obtenidos ocuparán la parte central del trabajo (apartado 4). Finalmente, una reflexión sobre el estado actual del tema y las futuras líneas de investigación cerrará este estudio (apartado 5).

\section{Género y humor en el monólogo humorístico}

La fundamentación teórica de este trabajo refuerza la idea de que el género es una variable social sujeta a razones contextuales, discursivas y psicológicas. De modo que la fluidez de esta categoría permite que se pueda construir y deconstruir a través del discurso (Bing \& Scheibman, 2014). Como veremos a continuación, el monólogo humorístico, como discurso planificado, es uno de los géneros en los que más claramente se observa la representación de la identidad de género.

Los primeros trabajos sobre el discurso desde la perspectiva de género surgieron durante la segunda ola del feminismo, más concretamente, tras la publicación en 1975 de la obra de Robin Lakoff, Language and woman's place. Además, con dicha obra se gestó la Teoría de déficit, que defendía que las mujeres carecían de poder, seguridad y fuerza en sus discursos. Posteriormente, en los 90, el estudio del discurso desde la perspectiva de género basado en el binomio mujer-hombre continuó con la Teoría de la diferencia, defendida por autoras como Deborah Tannen, cuyos trabajos como You just don't understand promovían la desigualdad de género y un análisis basado en la dicotomía de sexos. De modo que, para evitar esta dualidad, nuestra investigación se apoya en la Teoría constructivista del género, propuesta por la filósofa Judith Butler y que siguen lingüistas como West y Zimmerman (1987), Cameron (1998) o Crawford (2003). Según esta teoría, el género se entiende como una categoría social dinámica que se construye y deconstruye por medio de nuestras acciones, nuestro comportamiento y nuestro lenguaje (Butler, 1990, 1993). Por consiguiente, la teoría constructivista nos servirá de pilar teórico para el análisis de la construcción y deconstrucción del género a través de los estilos de habla empleados por las humoristas en el discurso directo.

\subsection{El estilo de habla marcado por el género en el discurso directo}

Como defendíamos en nuestra primera hipótesis, el lenguaje conforma nuestra visión del mundo y es uno de los principales medios que tenemos para expresar nuestros sentimientos e ideas. Esta visión ha sido apoyada por lingüistas como 
Crawford (2003: 1417), quien afirma que "language and speech style are important components of doing gender'. En esta línea se enmarcarían también las ideas de Deborah Cameron, las cuales quedan muy bien recogidas en la siguiente cita:

"If I talk like a woman this is not just the inevitable of the fact that I am a woman; it is one way I have of becoming a woman, producing myself as one. There is no such thing as practices ranging from the sort of work I do to my sexual preferences to the clothes I wear to the way I use the language" (Cameron, 1996: 46).

Los estudios sobre los estilos de habla en relación con la identidad de género surgieron a finales del siglo XX y fue, de hecho, Deborah Tannen quien distinguió el estilo de habla masculino y femenino por primera vez, bautizándolos como generolectos (Tannen, 1990). Sin embargo, se trata de una clasificación de los estilos de habla normotípicos que solo refleja el habla heteronormativa y presupone que estos van ligados al sexo del hablante (Stokoe \& Smithson, 2001). Este trabajo sigue las ideas propuestas por Gal (1995) y Cameron (1998), quienes piensan que el estilo de habla masculina y femenina no tienen por qué estar relacionados con el sexo biológico del hablante. De hecho, estos estilos de habla surgen a partir de los estereotipos y las normas culturales que la sociedad occidental ha fomentado en torno a la noción de masculinidad y feminidad heteronormativa. En esta línea, Ochs (1992) afirma que la identidad de género es una categoría dinámica que construimos con nuestros enunciados. Según el autor, no existe una identidad dada o fijada, sino que nosotros, como individuos, elegimos qué rasgos de nuestro género queremos mostrar a través del habla. Asimismo, Asch (1952) defiende que la identidad del hablante era un factor que afectar en la recepción de mensajes persuasivos por parte del interlocutor. Ello refuerza la idea de que los estilos de habla marcados por la identidad de género existen con independencia de nuestro sexo y somos nosotros, como sujetos, quienes decidimos cuál usar dependiendo del efecto comunicativo que deseemos lograr.

Estos estilos de habla marcados por el género son reconocibles en el discurso directo característico del monólogo humorístico. El estilo directo es un recurso empleado comúnmente en el monólogo humorístico, debido a la naturaleza polifónica de este tipo de discurso. Se trata de una estrategia narrativa que sirve para representar múltiples voces y recrear situaciones de forma verosímil, lo cual ayuda a su vez a involucrar al interlocutor (Tannen, 2007; Schwarz, 2009).

Siguiendo la teoría polifónica de la enunciación (Ducrot, 1986), en cada acto de habla, el humorista puede actuar como sujeto hablante, es decir, el individuo que pronuncia el enunciado; como locutor, que habla en primera persona, o como enunciador, cuando expresa ideas o puntos de vista de otras personas, que a menudo no comparte, esto es, no es responsable del acto ilocutivo. Mientras el oyente, en este caso el público se situará como sujeto empírico, locutor o destinatario ${ }^{4}$. Si bien, de acuerdo con Koven (2001) y Ruiz Gurillo (2006), el hablante transmite ya, 
inevitablemente, el mensaje desde su propia perspectiva y opinión. Así pues, pese a la multiplicidad de voces, la identidad de género que queda manifiesta en el estilo directo será aquella que reproduce la cómica.

Es evidente que el lenguaje humorístico empleado por las monologuistas será el reflejo de la identidad de género que se quiera representar para un determinado fin cómico y social. En el monólogo humorístico, el discurso directo está marcado por determinados elementos lingüísticos, prosódicos y kinésicos que contribuyen en el desencadenamiento del efecto humorístico (Ruiz Gurillo, 2013a) y que, además, pueden favorecer la subversión o el refuerzo de determinados estereotipos e ideologías asociadas al género. Por ello, cuando las humoristas emplean un estilo de habla que refleja, por medio de marcas acústico-melódicas y de indicadores humorísticos como la hipérbole, la metáfora o la polisemia (Ruiz Gurillo, 2014) una categoría de género estereotipada, esto puede deberse a su intención de criticar o burlar determinados clichés, o bien de imitar o representar dicha voz sin más pretensión. Para estudiar estas intenciones, examinaremos las elecciones lingüísticas de las cómicas como estrategias comunicativas para la construcción y representación del género dentro del monólogo humorístico.

\subsection{La construcción y deconstrucción de la identidad de género en el monólogo cómico}

El monólogo como discurso humorístico planificado es un buen ejemplo de género textual en el que se evidencia públicamente la construcción de la identidad, bien sea hegemónica o no normativa a través del refuerzo o la resistencia al statu quo. Las humoristas, de forma consciente o no, construyen su identidad de género sobre el escenario (Yus, 2002; Antoine, 2016). Es decir, la comediante enfatiza unos rasgos u otros de su personalidad en cada una de sus actuaciones, dependiendo de factores contextuales como la situación escénica, la temática del monólogo o del público asistente.

Es evidente que en la actualidad los escenarios se han convertido en una plataforma excelente para representar nuevas voces e identidades que transgreden lo establecido por la sociedad patriarcal. Partiendo del estudio de Greenbaum (1999), podemos afirmar que el contexto del stand-up promueve que las humoristas sean vistas como una autoridad en el escenario (ethos), por lo que tienen la capacidad de argumentar sus ideas sin generar demasiada controversia, debido al contexto humorístico en el que se enuncian. En esta línea, Yus (2012) describe al monologuista como una autoridad capaz de reforzar o desafiar determinadas representaciones culturales, como puedan ser los roles sexuales (Yus, 2002). No obstante, en la otra cara de la moneda, existe un tipo de humor que se encarga de mantener y reforzar la vieja jerarquía. Es aquel en el que los usuarios excusan sus afirmaciones bajo la broma o el chiste. En palabras de Weaver, Mora y Morgan (2016: 230): "there is always scope for 
denials of meaning (the 'it's just a joke' excuse)". De tal modo que el humor se convierte en una estrategia lingüística de la que nos servimos los seres humanos para definir nuestra identidad dentro de la sociedad y, así, ser parte o alejarnos de determinados grupos sociales.

\subsection{Los estilos humorísticos representados por las monologuistas}

Si sostenemos la hipótesis de que las humoristas construyen y deconstruyen su identidad de género por medio de una serie de patrones actitudinales y lingüísticos, esto se verá reflejado tanto en su estilo de habla como en su estilo humorístico. En este sentido, Tannen (1984: 130) afirma que "one of the most distinctive aspects of any person's style is the use of humor'.

Partimos de la clasificación de estilos propuesta en el Humor Styles Questionnaire por Martin et al. (2003) para poder analizar, gracias a las marcas e indicadores lingüísticos y extralingüísticos empleados en el discurso humorístico, qué estilos humorísticos muestran las monologuistas y qué relación tienen con sus estilos de habla y sus intenciones comunicativas. Distinguimos cuatro tipos de humor: agresivo, contraproducente, reafirmativo y afiliativo. Por un lado, diversos estudios han demostrado que es común en las mujeres producir un tipo de humor afiliativo con el que el interlocutor pueda empatizar y así crear lazos de unión con el endogrupo (Walker, 1998; Hay, 2001; Coates, 2003). En este caso, las monologuistas tratan temas de actualidad social con el que el público, principalmente femenino, se siente identificado. Por otro lado, el estilo de humor agresivo se suele emplear en el monólogo para mostrar el malestar y el enfado ante determinadas injusticias o hacia alguna persona o colectivo social como pueda ser la clase política, la iglesia o la sociedad patriarcal. Asimismo, el humor contraproducente o autodespreciativo es habitual en las monologuistas, ya que, al reírse de sus propias debilidades, desvirtúan el discurso machista y evitan que otros puedan emplear estos puntos de flaqueza como material de burla. Además, de acuerdo con Kotthoff (2006), este tipo de humor también contribuye a formar lazos de solidaridad. "Joking at one's own expense emphasizes commonalities, equality and group ties" (Kotthoff, 2006: 15). Finalmente, el estilo reafirmativo o de automejora muestra al hablante con una actitud positiva, de confianza en sí mismo, y enfatiza sus fortalezas y virtudes identitarias ante el oyente. 


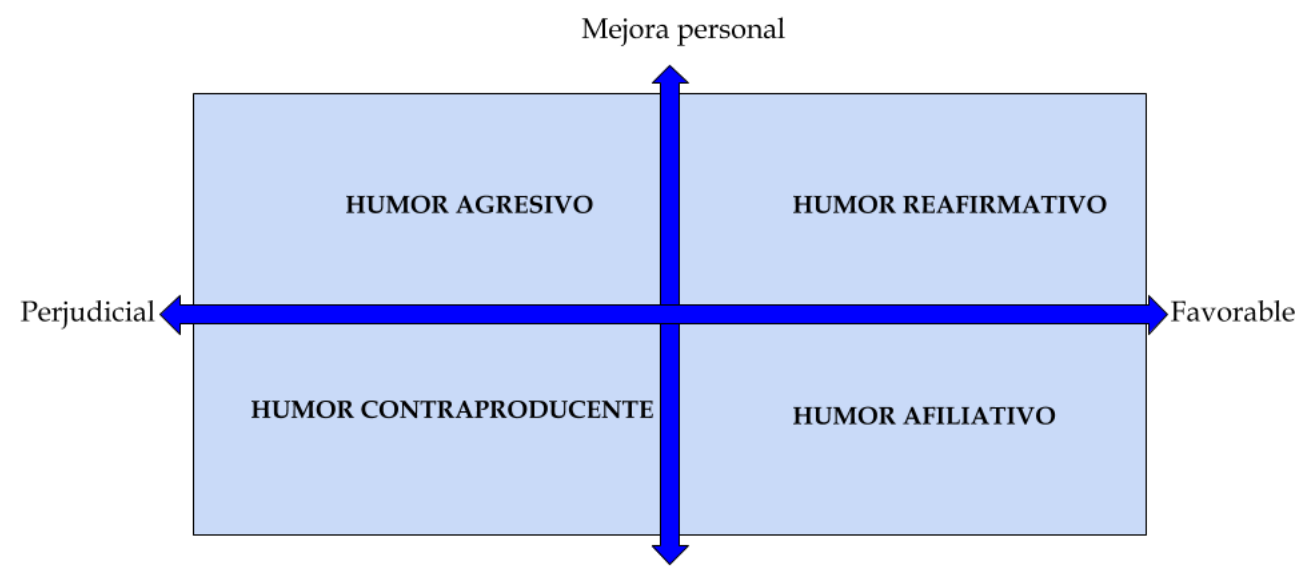

Mejora las relaciones sociales

Figura 1. Los cuatro estilos humorísticos descritos por Martin et al. (2003).

Ahora bien, cabe señalar que nuestro análisis de los estilos humorísticos de las informantes no se aplicará a su identidad personal, sino a la personalidad que adoptan como cómicas en el escenario, su stage persona (Greenbaum, 1999).

\section{Metodología y descripción del corpus}

Nuestra orientación metodológica busca cumplir con los objetivos propuestos, así como resolver las preguntas de investigación formuladas y corroborar las hipótesis planteadas en la introducción de este trabajo. Así pues, la metodología empleada para llevar a cabo esta investigación es de carácter hipotético-deductiva, lo que conlleva que sea, a la vez, exploratoria, descriptiva y contrastiva. En concreto, nos servimos de la transcripción y segmentación de las muestras humorísticas para extraer nuestros datos sobre la construcción discursiva de la identidad de género en el monólogo humorístico. Ello nos ha permitido estudiar el estilo de habla que emplean las cómicas durante la representación de su discurso, así como analizar los principales recursos lingüísticos y extra-lingüísticos que las humoristas utilizan para lograr con éxito su meta comunicativa.

\subsection{Datos del corpus analizado}

La muestra del presente estudio reúne un total de 10 monólogos, recogidos por la autora de este trabajo durante los años 2017 y 2018 en distintos locales y teatros del territorio español. Estos monólogos han sido representados por 10 mujeres humoristas del panorama de la comedia actual. En concreto, hemos analizado el discurso de las cómicas Eva Cabezas, Sil de Castro, Virginia Riezu, Patricia Sornosa, Patricia Espejo, Valeria Ros, Pamela Palenciano, Raquel Sastre, Coria Castillo y Susi Caramelo. En este sentido, cabe señalar que todas las cómicas analizadas tienen una amplia experiencia en el circuito de la comedia y muchas de ellas compaginan su faceta 
cómica con la de actriz o presentadora. Asimismo, proceden de diferentes puntos del país y la franja etaria se sitúa entre los 30 y 40 años de edad.

Cada uno de estos monólogos ha sido dividido en secuencias humorísticas, entendiéndose estas como una serie de intervenciones de la monologuista que giran en torno a un tema concreto y que son interrumpidas por las risas, aplausos e, incluso, comentarios del público (Val.Es.Co., 2014: 22-23). En este sentido, hemos transcrito, siguiendo el sistema de transcripción de Val.Es.Co. ${ }^{5} 284$ secuencias. Ello supone un total de 597 minutos -10 horas y 3 minutos de grabación- y 77946 palabras. Así pues, la cifra de humoristas y el tamaño del corpus han permitido recoger un número significativo de datos.

En la Tabla 1 se da cuenta de la duración, longitud en palabras y del número de secuencias de cada uno de los monólogos que constituyen el corpus en estudio.

Tabla 1. Datos de los monólogos.

\begin{tabular}{|l|c|c|c|}
\hline $\begin{array}{c}\text { Monólogo } \\
\text { representado por }\end{array}$ & Duración temporal & Número de palabras & $\begin{array}{c}\text { Número de } \\
\text { secuencias }\end{array}$ \\
\hline Coria Castillo & $31^{\prime} 08^{\prime \prime}$ & 2430 & 10 \\
\hline Pamela Palenciano & $90^{\prime}$ & 12612 & 35 \\
\hline Patricia Espejo & $80^{\prime}$ & 13083 & 40 \\
\hline Patricia Sornosa & $90^{\prime}$ & 10206 & 35 \\
\hline Raquel Sastre & $31^{\prime} 09^{\prime \prime}$ & 4699 & 25 \\
\hline Sil de Castro & $45^{\prime} 20^{\prime \prime}$ & 7002 & 24 \\
\hline Susi Caramelo & $37^{\prime}$ & 3955 & 33 \\
\hline Valeria Ros & $52^{\prime} 45^{\prime}$ & 8094 & 37 \\
\hline Virginia Riezu & $92^{\prime}$ & 11170 & 23 \\
\hline Eva Cabezas & $49^{\prime} 02^{\prime \prime}$ & 4695 & \\
\hline
\end{tabular}

En estas secuencias predomina el humor de carácter subversivo, es decir, nuestras informantes no buscan únicamente divertir al público y hacerle reír, sino que además se pretende visibilizar y denunciar problemas como el machismo o la desigualdad social. En particular, nos hemos centrado en aquellas secuencias en las que se emplea el discurso directo, en una o varias de las intervenciones. Esta elección viene motivada por nuestra intención de examinar las intervenciones en las que se observa un estilo de habla marcado por la identidad de género heteronormativa como estrategia para construir y deconstruir determinadas ideas asociadas a esta identidad.

\subsection{Materiales}

El principal instrumento para la obtención de datos fue la transcripción de los textos, siguiendo el sistema propuesto por el grupo Val.Es.Co. De esta forma, pudimos segmentar y etiquetar las secuencias en las que aparecía un estilo de habla marcado por la identidad de género. A raíz de los resultados obtenidos, elaboramos una hoja Excel en la que se indica el tema seleccionado, el objeto al que se dirige la 
burla o la crítica, elementos lingüísticos y extralingüísticos más señalados y el tipo de función que cumple el uso del estilo directo. A través de este programa, hemos diseñado las distintas tablas y gráficas con las que se ilustrarán nuestros resultados (apartado 3).

\section{Análisis de los resultados}

\subsection{Análisis cuantitativo: El discurso directo como estrategia discursiva de género}

Todas las monologuistas se sirven del discurso directo como estrategia narrativa en sus monólogos. De hecho, los resultados demuestran que el discurso directo aparece en 175 casos de las 284 secuencias transcritas, esto es, en un 61,6\% de los casos.

\section{Uso del discurso directo como estrategia humorística}

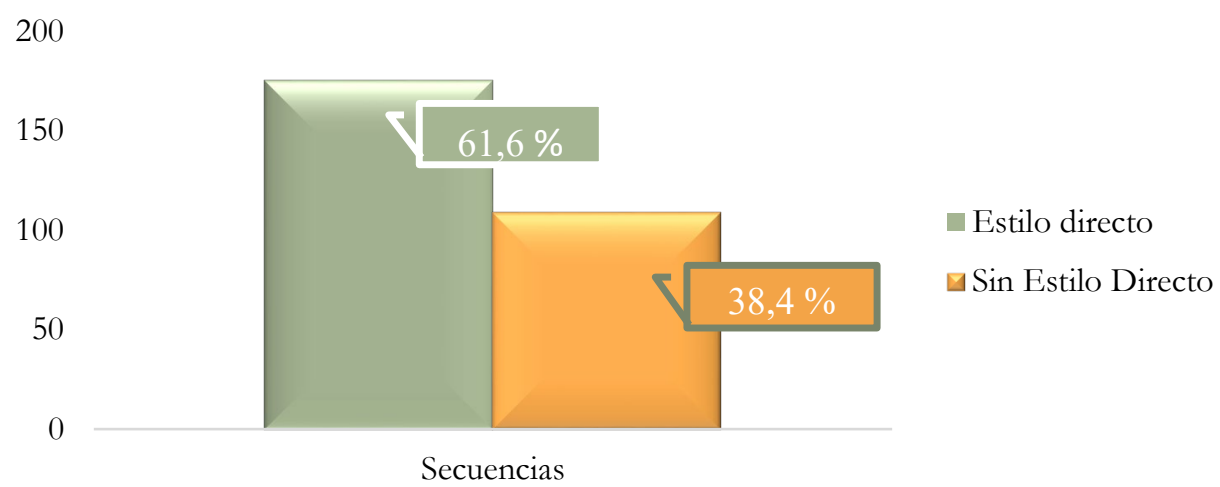

Gráfico 1. Uso del estilo directo en el corpus.

Asimismo, en la mayor parte de los casos en los que se emplea el discurso directo, este no viene marcado por el género. Así se observa cómo en el $57 \%$ de las 175 secuencias en las que se emplea el discurso directo, esta estrategia narrativa funciona como un recurso para hacer más verosímil la situación que se relata y para potenciar el efecto humorístico (Schwarz, 2009). Ahora bien, también se hace evidente que, en el $43 \%$ de los casos, este discurso directo está marcado por un estilo de habla que representa la identidad de género femenina o masculina heteronormativa a fin de conseguir un determinado efecto perlocutivo. De hecho, vemos como en el $17 \%$ de los casos el estilo directo refleja un estilo de habla marcado por la identidad de género cuyo efecto no es solo la risa o el divertimento del público, sino que también contribuye a mantener, e incluso reforzar, determinadas ideologías machistas de la sociedad patriarcal. Por otro lado, en el $26 \%$ de los extractos analizados de discurso directo, el estilo de habla promueve la ruptura de los roles sexuales y la subversión de las ideas heteronormativas asociadas al concepto de feminidad. Así pues, en la 
siguiente sección nos centraremos en analizar lingüísticamente cómo se consiguen estos efectos a través del discurso directo.

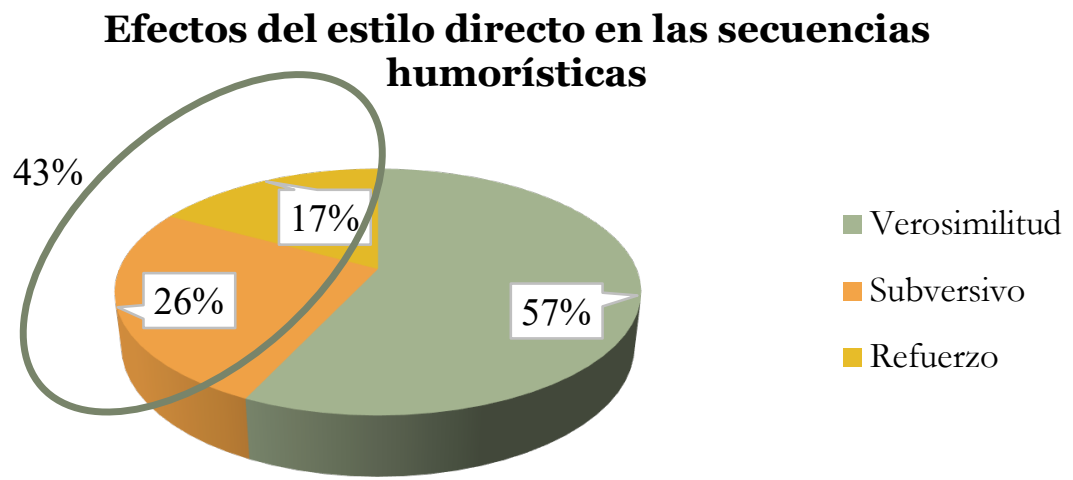

Gráfico 2. Porcentajes de los efectos en las secuencias en las que se emplea el discurso directo.

\subsection{Análisis cualitativo: El estilo directo como estrategia para el refuerzo y subversión de los roles sexuales}

El efecto cómico y la subversión o el refuerzo de los roles sexuales se produce gracias a las elecciones lingüísticas que toman las humoristas para su discurso (Ruiz Gurillo, 2016). Como demostraremos a través de los siguientes ejemplos, las cómicas recurren a diferentes marcas e indicadores humorísticos, así como a un estilo cómico determinado, como estrategias discursivas para representar la intención del hablante.

\subsubsection{Estilo de habla no marcado por el género. Función verosímil}

La secuencia que a continuación presentamos ilustra el principal uso del discurso directo en el monólogo humorístico, es decir, la verosimilitud. La monologuista representa un diálogo para poner en situación al oyente y hacer más creíble su discurso.

Ejemplo 1:

PATRICIA ESPEJO: leí que a un hombre lo que le gusta es encontrar a una mujer que tenga muchas cosas en común con él ¿vale?/ entonces el otro día fui a un

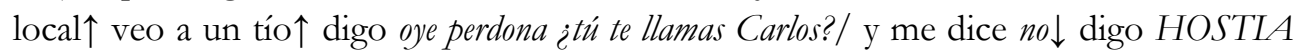
yo tampoco

PÚBLICO: RISAS

PATRICIA ESPEJO: digo ¿no me dirás que duermes por la noche con los ojos cerrados? PÚBLICO: RISAS

PATRICIA ESPEJO: dice ¿̇ú eres gilipollas?/ digo HOSTLA ¿tú también? 


\section{PÚBLICO: RISAS Y APLAUSOS}

PATRICIA ESPEJO: que-que el tío se ve que se rayó porque vio que teníamos muchas cosas en común $\rightarrow$

\section{PÚBLICO: RISAS}

Como ya hemos observado en el análisis cuantitativo de los resultados, el discurso directo funciona en el monólogo humorístico como un recurso para hacer más verosímil la situación que se relata y para potenciar el efecto cómico. De hecho, en este caso, no existe un estilo de habla marcado por el género cuando se representa la voz de los protagonistas del diálogo. En el ejemplo presentado, el discurso directo sirve como estrategia narrativa para promover el efecto humorístico, el cual se desencadena gracias al juego de palabras que la monologuista realiza con la expresión 'tener muchas cosas en común'. El hecho de llevar al extremo dicha expresión para promover la comicidad, así como la entonación empleada, denotan un estilo humorístico contraproducente o autodespreciativo en el que ella se sitúa como objeto de la risa.

\subsubsection{Estilo de habla masculino. Función subversiva}

En el siguiente fragmento observamos cómo la humorista manifiesta su identidad como mujer y desarrolla su discurso mostrando un estilo humorístico afiliativo con el sector femenino y, a la vez, agresivo con el masculino ${ }^{6}$. Además, la cómica emplea el estilo directo y los rasgos de la voz masculina estereotipada para recrear una situación común de la sociedad patriarcal, como es la presión social impuesta a las mujeres para ser madres.

Ejemplo 2:

PATRICIA SORNOSA: bueno si como yo eres ya una mujer/ tienes 40 años-que tengo yoo y aún no tienes HIJOS// cualquiera-pero cualquiera ¿eh? CUALQUIERA se cree en el derecho de decirte esa frase tan asquerosa// pos se te va a pasar el arroz.

PÚBLICO: RISAS

PATRICIA SORNOSA: ¿se te va a pasar el arroz? ¿pero se puede ser más ASQUEROSAMENTE MACHISTA? / se te va a pasar el arroz $\rightarrow$ ijoder! ¿Pero es que hasta en las frases hechas nos tenéis que poner a cocinar?

PÚBLICO: RISAS

PATRICIA SORNOSA: que no sólo es que te pongan a cocinar/ es que no se fían que lo hagas bien y se quedan detrás vigilando ¿eh?/ y te dan instrucciones ((y te dicen)) estate atenta abi $\downarrow$ estate atenta que se te va a pasar el arroz

PÚBLICO: RISAS

PATRICIA SORNOSA: que yo no he hecho arroz EN MI PUTA VIDA/ pero quee sé yo que se te va a pasar $\rightarrow$ baz algo/ HAZ ALGO/ mmuévelo/ fóllatelo/ haz algo con el arroz.

PÚBLICO: RISAS 
En el ejemplo vemos una ruptura de las expectativas, puesto que la monologuista desafía al statu quo y muestra su indignación ante el pensamiento machista sobre la edad idónea de la mujer para ser madre a través del uso de preguntas retóricas y los adverbios y adjetivos valorativos empleados. Aunque, sin duda, es el uso de la polifonía y de la desautomatización de la unidad fraseológica 'se te va a pasar el arroz', enunciada por el locutor masculino, lo que desencadena las carcajadas del público y la ruptura del rol sexual de la mujer como madre.

De igual forma, en la tercera secuencia, que pasamos a comentar a continuación, la monologuista emplea el estilo de habla masculino para situar como objeto de burla a aquellos maltratadores que se disfrazan de maltratados. Observamos cómo emplea un estilo de humor afiliativo con las mujeres que han sufrido violencia mientras a su vez realiza una crítica hacia el discurso victimista de los agresores.

Ejemplo 3:

PAMELA PALENCIANO: esa se ve de las que pegaan $\rightarrow$ / al Antonio tiene que tenerlo fino/ eh no os riais ¿vale? que esto es muy serio tío que yo soy un tío maltratao ¿vale? 7

PÚBLICO: RISAS

PAMELA PALENCIANO: la gente se rie pero existimos $\downarrow$ el hashtag existe/ búscalo PÚBLICO: RISAS

PAMELA PALENCIANO: estamos organizados en Forocoches/ lo máximo PÚBLICO: RISAS

PAMELA PALENCIANO: yo sé que la gente se ríe pero yo sufro un montón ¿sabes?/ habemos hombres que sufrimos violencia/ seremos pocos pero somos/ ¿sabes?

\section{PÚBLICO: RISAS}

El humor de esta secuencia se origina con la contradicción irónica del hombre maltratador que se confiesa maltratado. Por otra parte, el registro dialógico empleado es una técnica argumentativa que ayuda al monologuista a conectar con el público y convencer de su discurso (Ruiz Gurillo, 2013b). El estilo de habla masculino empleado en el discurso directo incorpora un lenguaje humorístico que promueve la desarticulación del poder del agresor y lo sitúa como objeto de burla. Así, el uso de expresiones coloquiales, las repeticiones y el eco a referencias socioculturales como Forocoches o Twitter desencadenan las risas del público. Asimismo, los distintos elementos prosódicos, es decir, la entonación, el tono, las pausas, el énfasis, etc., contribuyen a reproducir la identidad masculina y ayudan al oyente a inferir el humor y la actitud del hablante. También, otra clave del éxito en la representación de la identidad masculina son las marcas kinésicas, esto es, las expresiones faciales y movimientos corporales que realiza Pamela Palenciano mientras recrea el diálogo. 
En estos dos casos en los que se emplea el estilo de habla masculino -directo, autoritario y con una tonalidad de vOz grave-, este sirve como estrategia humorística para ejecutar la broma o la burla hacía los hombres. Además, este estilo de habla, junto con los movimientos y gestos corporales, ayuda a romper con las limitaciones impuestas a la identidad femenina.

\subsubsection{El estilo de habla femenino. Función de refuerzo y subversión}

Por otro lado, como muestran los resultados cuantitativos comentados en la sección previa, el discurso directo marcado por la identidad de género puede no solo servir para romper con los roles sexuales, sino que también puede contribuir a reforzarlos y perpetuarlos. El siguiente ejemplo ilustra este fenómeno. Se observa cómo la monologuista recurre a un chiste enlatado sobre el tópico de que las mujeres rubias son tontas para generar la risa del público allí presente.

Ejemplo 4:

SIL DE CASTRO: a mí por ejemplo me han gustado mucho de siempre-ee es un tópico ¿eh? yo lo sé $\downarrow$ (0) los chistes de rubias/ me hacen mucha gracia sí// hola ¿qué tal? ¿cómo estás cari? túu túu eres teñida ¿no?

PÚBLICO: RISAS

\section{(...)}

SIL DE CASTRO: ahora sí que está insultándome pero como para por dentro/¿cómo te llamas $\downarrow$ cari?

\section{SEÑORA DEL PÚBLICO: Teo}

SIL DE CASTRO: uy qué nombre más bonito/ bueno pues dicen que estaba un día Teo con su descapotable-que ella tiene un descapotable pero no lo saca por Rafaelbunyol porque sabemos cómo está el patio ¿eh?/ entonces estaba ella un día con su descapotable y conduciendo así a lo loco- porque conduce bastante mal/ ella estaba (0) liándola parda y entonces adelantó a un camionero mal/ y el camionero se enfadó muchísimo y entonces ¡AAH! la puso contra la cuneta $\downarrow$ se bajo del camión $\downarrow$ se fue hacia ella $\downarrow$ la cogió con los pelos y la bajó del coche/ ¿te acuerdas qué mal lo pasaste?/ bueno la baja del coche y el camionero coge una tiza/ hace un círculo en el suelo y le dice mira rubia de ahí no te mueves hasta que yo lo diga/ se queda Teo acojonada dentro del círculo ¿no? viendo cómo el camionero coge un bate de béisbol se va para el descapotable y empieza PAA PAA y le revienta toda la luna del coche/ cuando se gira estaba Teo dentro del círculo así ja ja ja ja y el tío flipando diciendo pero ¿de qué se está riendo esta tía? si le estoy reventando el coche $\downarrow$ te vas a enterar $\uparrow$ hombre ya/ pilla una navaja y empieza jigg y le raja toda la tapicería de cuero/ cuando se gira estaba Teo así

\section{PÚBLICO: RISAS}

SIL DE CASTRO: y el tío flipando en plan pero ¿de qué se ríe esta tía? te vas a enterar hombre/ pilla un bidón de gasolina $\uparrow$ rocía el coche y le pega fuego/ cuando se 
gira pero en el suelo (0) AAH QUE ME PARTO ME PARTO/dice el camionero pero vamos a ver rubia ¿de qué coño te ríes?/ dicee $\rightarrow$ es que cuando no me mirabas me he salido del círculo CUATRO VECES

\section{PÚBLICO: RISAS Y APLAUSOS}

En este caso, la cómica elige a una señora rubia del público como personaje principal de la historia para captar la atención de los oyentes y así recrear de manera más personal un diálogo protagonizado por ella como locutora junto con un camionero. De modo que la polifonía sirve en este caso para que la monologuista reproduzca el estilo de habla tanto masculino como femenino. En el caso de la identidad femenina, esta se representa sobre todo durante el remate final del chiste, pues es aquí donde la cómica hace uso de un tono más agudo y del habla entre risas para imitar este estilo de habla estereotipado. Además de reforzar el tópico, este chiste genera las risas y los aplausos del público, pues narra una situación incongruente debido al sinsentido de que a la protagonista le importe más el haber desobedecido las órdenes del camionero que los desperfectos de su vehículo. Este tipo de humor sería principalmente contraproducente, ya que ataca a su propio endogrupo y refuerza los prejuicios sobre las mujeres de cabello rubio.

En el caso contrario se situaría el siguiente fragmento. En esta secuencia también se emplea el estilo de habla femenino estereotipado, pero para un fin distinto: la burla. La monologuista emplea el discurso directo para reproducir el habla de las pijas de Murcia y muestra una actitud de reprobación ante sus preocupaciones banales como los cotilleos o el aspecto físico.

Ejemplo 5:

RAQUEL SASTRE: pijas que te las encuentras en medio de la Gran Vía de Murcia y te dicen jo tía qué fuertee que fuerte ¿te acuerdas de Borja? Pues me acaba de contar una cosa superfuerte/ espérate que me llama mi mamuchi por teléfono/ acha mama dime

\section{PÚBLICO: RISAS}

RAQUEL SASTRE: pijas de toda la vida y además van todas a escuela de modelos/ y ya te las ves tú llegar ahí superpijas pero pijas pijas pijas pijas de verdad/ pijas que les haces un cunilingüe y te sabe a rodaballo sobre lecho de setas con emulsión de Pedro Ximénez

\section{PÚBLICO: RISAS}

RAQUEL SASTRE: y van todas a escuela de modelos/ el otro día me encuentro a una de ellas por la calle y me dice jo tía vengo supercansada de la escuela de modelos/ y yo bff vuela puta

\section{PÚBLICO: RISAS}

RAQUEL SASTRE: dice tía tú has entrado alguna vez a una escuela de modelos? Abi no si no cabes por la puerta / y yo ¿y a que te pongo una multiplicación y lloras?

\section{PÚBLICO: RISAS}


La humorista logra con éxito su objetivo comunicativo, la burla hacia este tipo de feminidad, a través de diferentes indicadores y marcas humorísticas. Por un lado, con prefijos como 'super' o sufijos como uchi, la repetición de la expresión 'qué fuerte' y la entonación del habla estereotipada de mujer pija contrastan con la expresión coloquial propia de la región de Murcia 'acha'. Este cambio de registro refleja la burla y desencadena el humor. Asimismo, la humorista emplea un humor agresivo con el que intenta deconstruir la identidad de género hegemónica. Ello se aprecia en el lenguaje que selecciona al actuar como locutora. El uso de hipérboles y adjetivos peyorativos evidencian la agresividad propia de este estilo humorístico.

En definitiva, hemos apreciado cómo el discurso directo, además de servir para fomentar la verosimilitud y la atención del público, puede generar otros efectos relacionados con la construcción y deconstrucción del género. A través del monólogo, las humoristas consiguen cuestionar las estructuras sociales y visibilizar injusticias con el fin de desmitificar los roles sexuales que giran en torno a la figura de la mujer y deconstruir así la identidad femenina impuesta por el patriarcado. No obstante, por otro lado, este discurso directo marcado por el estilo de habla femenino o masculino puede tener también efectos adversos con los que, de forma inconsciente o no, las humoristas refuerzan determinados estereotipos de género y perpetúan la estructura hegemónica. Asimismo, en cuanto a los estilos humorísticos observados en este tipo de discurso planificado, hemos visto que las cómicas no solo intentan crear lazos de solidaridad con el público femenino a través de un estilo afiliativo y/o autodespreciativo, sino que también usan su autoridad sobre el escenario para reivindicar su identidad de género y deconstruir los prejuicios y roles impuestos por el patriarcado. En otras palabras, lo que hacen es reconstruir su identidad de género. De acuerdo con Gilbert (2004), la actuación sobre el escenario empodera a la comediante para desafiar y revelarse ante la hegemonía patriarcal. Cuando las humoristas critican y desafían el discurso patriarcal, están construyendo su identidad de género a la vez que deconstruyendo aquella asignada por los convencionalismos de la sociedad en la que vivimos.

\section{CONCLUSIONES}

Este estudio nos ha permitido comprobar la multifuncionalidad del estilo directo como estrategia narrativa en el monólogo humorístico. Así pues, se ha demostrado que, en ocasiones, el estilo directo empleado en el monologo humorístico refleja estilos de habla marcados por la identidad de género masculina y femenina heteronormativa, que colaboran no solo en el desencadenamiento del efecto humorístico, sino también en la subversión o refuerzo de los roles sexuales. De hecho, si bien es cierto que el humor juega un papel importante en la desarticulación del statu quo de género y en la subversión de la identidad heteronormativa femenina, llena de estereotipos y clichés, este también contribuye a reforzar y perpetuar determinados roles sexuales. 
Asimismo, se confirma nuestra hipótesis de partida de que el lenguaje humorístico empleado por las cómicas es el reflejo de la construcción de la identidad de género, que se representa en el monólogo como discurso humorístico planificado. Los ejemplos presentados en el presente trabajo son la mejor prueba de que hay una nueva línea en este género humorístico que se enfrenta al patriarcado y traspasa los límites del discurso hegemónico. Además, el análisis de los datos nos ha permitido también corroborar que el monólogo humorístico supone un medio excelente para examinar la mutabilidad del género, la combinación discursiva de las identidades y el reflejo de estas en el discurso.

Ahora bien, si consideramos que el género es una variable que afecta en gran medida a nuestra identidad social, junto con otros factores condicionantes como la etnia, la edad o la clase social, sería también interesante, para futuras investigaciones, analizar este tipo de discurso humorístico en otras culturas. De hecho, mientras que el monólogo alternativo y reivindicativo está todavía en un estado primigenio dentro de la comedia española y su difusión es todavía limitada en determinados contextos, el humor desafiante y subversivo tiene una larga tradición en Estados Unidos. En el stand-up comedy estadounidense se suelen tratar sin tapujos temas como el racismo, la homofobia o el machismo, ya que resulta más sencillo tratar asuntos controvertidos en un contexto humorístico y distendido, como el que genera el monólogo, que a través de cualquier otro género serio (Chiaro \& Baccolini, 2014).

Del mismo modo, si concebimos el género como un factor que incide en el habla y se construye en el habla, consideramos que habría que realizar estudios más amplios sobre la construcción de este fenómeno en otros géneros humorísticos. Sin duda, se necesitaría un estudio empírico más amplio que aborde este tema desde el paradigma cuantitativo, para poder realizar afirmaciones de carácter más general.

\section{REFERENCIAS BIBLIOGRÁFICAS}

Antoine, K. (2016). Pushing the Edge'of Race and Gender Hegemonies through Stand-up Comedy: Performing Slavery as Anti-racist Critique. Etnofoor, 28(1), $35-54$.

Asch, S. E. (1952). Social Psychology. Englewood Cliffs, NJ: Prentice-Hall.

Beauvoir, S. (1949). El segundo sexo. Madrid: Cátedra.

Bing, J. \& Scheibman, J. (2014). Blended Space as Subversive Feminist Humor. En D. Chiaro \& R. Baccolini (Eds.), Gender and Humor: Interdisciplinary and International Perspectives (pp. 13-29). Londres: Routledge,Taylor \& Francis.

Boxer, D. \& Cortés-Conde, F. (1997). From bonding to biting: Conversational joking and identity display. Journal of Pragmatics, 27(3), 275-294. 
Briz, A. \& Grupo Val.Es.Co. (2002). Corpus de conversaciones coloquiales. Madrid: Arco/Libros.

Butler, J. (1990). Gender trouble: Feminism and the subversion of identity. Nueva York: Routledge.

Butler, J. (1993). Critically queer. GLQ: A journal of Lesbian and Gay Studies, 1(1), 17-32.

Cameron, D. (1996). Style policy and style politics: A neglected aspect of the language of the news. Media, Culture \& Society, 18(2), 315-333.

Cameron, D. (1998). Performing gender identity. Language and gender: a reader. Malden, MA: Blackwell.

Chiaro, D. \& Baccolini, R. (Eds.). (2014). Gender and humor: Interdisciplinary and international perspectives (Vol. 63). Londres: Routledge.

Coates, J. (2003). Men talk: Stories in the making of masculinities. Oxford: Blackwell.

Crawford, M. (2003). Gender and humor in social context. Journal of pragmatics, 35(9), 1413-1430.

Ducrot, O. (1986). Esbozo de una teoría polifónica de la enunciación. El deciry lo dicho. Polifonía de la enunciación, 175-238.

Gal, S. (1995). Language, gender, and power. And Anthropological Review. En K. Hall \& M. Bucholtz (Eds.), Gender articulated: Language and the socially constructed self (pp. 169-182). Nueva York: Routledge.

Gilbert, J. R. (2004). Performing marginality: Humor, gender, and cultural critique. Detroit, Michigan: Wayne State University Press.

Goffman, E. (1977). The arrangement between the sexes. Theory and society, 4(3), 301331.

Goffman, E. (1981). Forms of talk. Pennsylvania: University of Pennsylvania Press.

Greenbaum, A. (1999). Stand-up comedy as rhetorical argument: An investigation of comic culture. Humor-International Journal of Humor Research, 12(1), 33-46.

Grupo Val.Es.Co. (2014). Las unidades del discurso oral. La propuesta Val.Es.Co. de segmentación de la conversación (coloquial). Estudios de Lingüistica del Español, 35, 13-73.

Hay, J. (2001). The pragmatics of humor support. Humor - International Journal of Humor Research, 14(1), 55-82 [en línea]. Disponible en: https://doi.org/10.1515/humr.14.1.55. 
Hall, K. (1995). Lip service on the fantasy lines. En K. Hall \& M. Bucholtz (Eds.), Gender Articulated: Language and the Socially Constructed Self (pp. 183-216). Nueva York: Routledge.

Kotthoff, H. (2006). Gender and humor: The state of the art. Journal of pragmatics, 38(1), 4-25.

Koven, M. (2001). Comparing bilinguals' quoted performances of self and others in tellings of the same experience in two languages. Language in society, 30(4), 513558.

Lakoff, R. (1975). Language and woman's place. Nueva York: Harper \& Row.

Martin, R. A., Puhlik-Doris, P., Larsen, G., Gray, J. \& Weir, K. (2003). Individual differences in uses of humor and their relation to psychological well-being: Development of the Humor Styles Questionnaire. Journal of research in personality, 37(1), 48-75.

Ochs, E. (1992). Indexing gender. Retbinking context: Language as an interactive phenomenon, 11, 335-358.

Ruiz Gurillo, L. (2006). Hechos pragmáticos del español. Alicante: Publicaciones de la Universidad de Alicante.

Ruiz Gurillo, L. (2012). La lingüistica del humor en español. Madrid: Arco Libros.

Ruiz Gurillo, L. (2013a). El monólogo humorístico como tipo de discurso. El dinamismo de los rasgos primarios. Cuadernos AISPI: Estudios de lenguas $y$ literaturas hispánicas, 2, 195-218.

Ruiz Gurillo, L. (2013b). Eva Hache y El Club de la Comedia: Del guion monológico al registro dialógico. Onomázein: Revista de lingüistica, filología y traducción de la Pontificia Universidad Católica de Chile, 28, 148-161.

Ruiz Gurillo, L. (2014). Infiriendo el humor. Un modelo de análisis para el español. Revista CLAC, 59, 148-162 [en línea]. Disponible en: http://pendientedemigracion.ucm.es/info/circulo/no59/rgurillo.pdf

Ruiz Gurillo, L. (2016). Metapragmatics of humor: Variability, negotiability and adaptability in humorous monologues. En L. Ruiz Gurillo (Ed.), Metapragmatics of bumor: Current research trends (pp. 79-101). Amsterdam/Filadelfia: John Benjamins.

Schwarz, J. (2009). Linguistic aspects of verbal humor in stand-up comedy. Thesis dissertation. [en línea]. Disponible en: https://publikationen.sulb.unisaarland.de/handle/20.500.11880/23601 
Stokoe, E. H. \& Smithson, J. (2001). Making gender relevant: Conversation analysis and gender categories in interaction. Discourse \& Society, 12(2), 217-244.

Tannen, D. (1984). Conversational style: Analyring talk among friends. Oxford: Oxford University Press.

Tannen, D. (1990). You just don't understand (Vol. 22). Nueva York: Ballantine Books.

Tannen, D. (2007). Talking voices: Repetition, dialogue, and imagery in conversational discourse (Vol. 26). Cambridge: Cambridge University Press.

Walker, N. A. (1988). A very serious thing: Women's bumor and American culture (Vol. 2). Minnesota: U of Minnesota Press.

Weaver, S., Mora, R. A. \& Morgan, K. (2016). Gender and humour: Examining discourses of hegemony and resistance. Social semiotics, 26(3), 227-233

West, C. \& Zimmerman, D. H. (1987). Doing gender. Gender and Society, 1(2), 125-151.

Wittgenstein, L. (1953). Investigaciones filosóficas (Philosophische Untersuchungen). Londres: Kegan Paul.

Yus, F. (2002). Stand-up comedy and cultural spread: The case of sex roles. Babel AFLAL, 10, 245-292.

\section{NOTAS}

${ }^{1}$ Esta investigación se enmarca en el Proyecto Humor de género: Observatorio de la identidad de mujeres y hombres a través del humor (PROMETEO/2016/052) (Generalitat Valenciana, 2016-2019). Para más datos sobre el grupo de investigación GRIALE, consultar [en línea]. Disponible: http://dfelg.ua.es/griale/.

2 A principios del siglo XX, Wittgestein (1953) reconoció la relación entre el lenguaje y la actividad social, afirmando que el significado estaba ligado a su uso.

${ }^{3}$ Esta idea del género como constructo social fue tomada de la escritora Simone de Beauvoir (1949) cuando dijo en su obra El segundo sexo que 'no se nace mujer: llega una a serlo'.

${ }^{4}$ Existen diferentes teorías que intentan explicar la función de la multiplicidad de voces en el discurso. Para Goffman (1977, 1981), el hablante y el oyente son agentes sociales. El sujeto puede actuar como autor, responsable de lo dicho, animador, enunciando palabras ajenas, o representante, es decir como portavoz de un colectivo; y el oyente hace el papel de público, auditorio o audiencia.

5 Pueden consultarse las claves del sistema de transcripción Val.Es.Co. en Briz y Grupo Val.Es.Co. (2002: 28-38) o [en línea]. Disponible: https://www.uv.es/valesco/sistema.pdf 
${ }^{6}$ Boxer y Cortés-Conde (1997) definen este tipo de humor como bonding and biting.

${ }^{7}$ Camina con las piernas abiertas y expresa falsa tristeza en su rostro. 